\title{
CTL to HIV-1: Surrogates or sirens
}

\section{The generation of cytotoxic T lymphocyte response may be an essential part of an effective immune response to HIV infection. It may also do more harm than good (pages 330-336).}

The recognition that cytotoxic $T$ lymphocytes (CTL) might be an important surrogate marker or correlate for immune protection against HIV-1 infection or disease has gradually evolved. Several observations have supported the incorporation of CTL induction as a goal in HIV-1 vaccine design. First, CD8+, MHC class Irestricted CTL can be a powerful tool in the host's armamentarium to blunt the effects of a number of viral diseases (reviewed in Koenig et al. ${ }^{1}$, see p. 329 of this issue of Nature Medicine). Recently, a few infected persons ${ }^{2,3}$ (and Connick, E. et al., manuscript submitted) and monkeys ${ }^{4}$ have been documented to have detectable precursor CTL at the time of initial HIV-1 or SIV clearance. However, detectable neutralizing antibody to autologous or other primary HIV isolates from the same individuals is not observed until substantially later, if at all ${ }^{2}$. Second, a few, apparently uninfected infants born to HIV-infected mothers ${ }^{5-7}$ and some seronegative subjects with high risk of sexual exposure to $\mathrm{HIV}^{8,9}$ (that is, exposed but "uninfected" subjects) have been reported to have CTL directed against selected epitopes of HIV-1 proteins. Finally, information from studies of long-term survivors with high CD4+ T lymphocyte counts suggests that CTL may function by limiting virus spread or replication ${ }^{10,11}$ reviewed in Schrager et al. ${ }^{12}$ (Rinaldo, C.R. et al., Harrer, T. et al. and Riviere, Y. et al. manuscripts submitted).

In general, functionally active $\mathrm{CD} 8^{+}$ CTL to infectious viruses can be generated quickly de novo. In HIV or SIV infections, this appears to be within days to a few weeks ${ }^{2,4}$. In addition, HIV-specific CTL activity can be detected in the peripheral circulation, without in vitro stimulation, in most HIV-infected subjects ${ }^{13,14}$. A quiescent memory/precursor population can be stored in lymphoid tissues as a CTL 'reserve' for rapid recall. When functionally active, $\mathrm{CD} 8^{+} \mathrm{CTL}$ produce a substantial cytokine response that can profoundly depress viral replication for many viruses. HIV-specific CTL clones have been shown to produce interferon gamma and several other antiviral cytokines ${ }^{15}$. However, in an HIV-1-infected person, these cytokines may provide

\section{BONNIE J. MATHIESON}

stimulatory signals for viral replication. Koenig et al. ${ }^{1}$ observed a brief viral increase, despite the apparently successful delivery of the therapy.

To induce CTL, vaccine strategies must introduce viral protein epitopes into the MHC class I antigen-processing pathway. In addition to infection with the virus itself or even an attenuated virus ${ }^{16}$, AIDS vaccines designed with live, replicating viral or bacterial vectors, such as vaccinia $^{17,18}, \mathrm{BCG}^{19,20}$, or Salmonella have been reported to induce CTL. Non-replicating viral vectors, such as canary pox (ALVAC) have also been reported to induce CTL (Francini, G. et al. and Riviere, Y. et al., personal communication). In addition, some adjuvants that enable antigen to enter cytoplasmic pathways have enabled CTL induction to AIDS virus antigens $^{21,22}$. Finally, recent studies with nucleic acid vaccines indicate that CTL can be generated by this strategy ${ }^{23}$ (Robinson, H. et al., personal communication). Virtually all of these vaccine strategies that exhibited at least memory/precursor CTL have already failed to protect animals unequivocally (data presented by the cited groups of investigators at the Conference on AIDS Vaccine Development, November 6-10, 1994).

Somewhere in the rush to design HIV-1 vaccines that would induce CTL, the mode of action was left in the shadows, that is, CTL kill MHC class I-matched infected cells. They could never prevent infection unless they were (1) in an effector state of stimulation at the time of viral challenge, (2) at the site of infection, and most critically, (3) matched for MHC class I. Thus the question is as follows: How rapidly can virus-specific CTL come into play in a 'CTL' vaccine-primed host compared to either a "T helper (Th)" primed host or a totally naive host? Will a delay for de novo generation of CTL effectors draw the host onto rocky shores? Can this be ameliorated by some antibody that slows down the virus?

Further, as Koenig et al. ${ }^{\prime}$ illustrate, there is concern that CTL can contribute to the pathogenesis of HIV-1 disease when the balance is tipped in favour of an already widely dispersed viral pathogen. The balance in favour of the host is further compromised, when there is a highly focused, possibly unique, immune respons $\mathrm{e}^{1}-$ in this case the cloned CTL. The virus then only has to evade a single 'enemy' by simple variation, which HIV-1 has repeatedly demonstrated is its forte for monovalent drug therapy. Because the range of CTL recognition is tightly controlled by an individual's MHC genes, even when responses are successfully induced by virus infection, CTL may use only one T cell receptor beta family, or be limited to only one or two epitopes on a single viral pro$\operatorname{tein}^{24}$.

Thus, clonal CTL, as therapies, may be like mythical Sirens, offering the ability to lyse virus-infected cells at the cost of the host's cells. The solution may lie in inducing an increased breadth of the immune response (as proposed by others ${ }^{14}$ ), increasing the quality of the response (that is, the precise specificity), or increasing the height of the response. The latter is problematic because data on attenuated viruses indicate that more replication produces better $\mathrm{CTL}^{16}$.

1. Koenig, S. et al. Transfer of HIV-1-specific cytotoxic T lymphocytes to an AIDS patient leads to selection for mutant HIV variants and subsequent disease progression. Nature Med. 1, 330-336 (1995)

2. Koup, R. et al. Temporal association of cellular responses with the initial control of viremia in primary HIV-1 syndrome. I. Virol. 68, 4650-4655 (1994).

3. Borrow, P., Lewicki, H., Hahn, B. H., Shaw, G. M \& Oldstone, M. B. Virus-specific CD8+ cytotoxic T-lymphocyte activity associated with control of viremia in primary human immunodeficiency virus type 1 infection. J. Virol. 68, 6103-6110 (1994).

4. Yasutomi, Y., Reiman, K., Lord, C., Miller, M. \& Letvin, N. Simian immunodeficiency virus-specific CD8 + lymphocyte response in acutely infected rhesus monkeys. J. Virol. 67, 1707-1711 (1993).

5. Cheynier, R. et al. Cytotoxic T lymphocyte responses in the peripheral blood of children born to HIV-1-infected mothers. Eur. J. Immunol. 22 , 2211-2217 (1992).

6. Rowland-Jones, S. L. et al. HIV-specific CTL activity in an HIV-exposed but uninfected infant. Lancet 341, 860-861 (1993).

7. Aldhous, M.C., Watret, K. C., Mok, J. Y., Bird, A G. \& Froebel, K. S. Cytotoxic T lymphocyte activity and CD8 subpopulations in children at risk of HIV infection. Clin. exp. Immunol. 97 61-67 (1994).

8. Langlade-Demoyen, P., Ngo-Giang-Huong, N., 
Ferchal, F. \& Oksenhendler E. HIV nef-specific cytotoxic T lymphocytes in noninfected heterosexual contacts of HIV-infected patients. J. clin. Investig. 93, 1293-1297 (1994).

9. Rowland-Jones, S. et al. HIV-specific cytotoxic T-cells in HIV-exposed but uninfected Gambian women. Nature Med. 1, 59-64 (1995).

10. Johnson, R. P., Trocha, A., Buchanan, D. M. \& Walker, B. D. Identification of overlapping HLA class J-restricted cytotoxic $\mathrm{T}$ cell epitopes in a conserved region of the human immunodeficiency virus type 1 envelope glycoprotein: Definition of minimum epitopes and analysis of the effects of sequence variation. J. exp. Med. 175, 961-971 (1992).

11. Keet, I.P.M. et al. Characteristics of long-term asymptomatic infection with human immunodeficiency virus type 1 in men with normal and low CD4+ cell counts. I. infect. Dis. 169, 1236-1243 (1994).

12. Schrager, L. K., Young, J.M., Fowler, M.G., Mathieson, B.J. \& Vermund, S. H. Long-term survivors of HIV-1 infection: definitions and research challenges. AIDS 8 (suppl 1), S95-S108 (1994).

13. Gotch, F.M., Nixon, D.F., Alp, N., McMichael A.J. \& Borysiewicz, L.K. High frequency of memory and effector gag-specific cytotoxic T lymphocyte in HIV seropositive individuals. Intl. Immun. 2, 707-712 (1990).

14. Lieberman, J. et al. Cytotoxic T lymphocytes from HIV-1 seropositive individuals recognize immunodominant epitopes in gp 160 and reverse transcriptase. J. Immun. 148, 2738 (1992).

15. Jassoy, C. et al. Human immunodeficiency virus type 1-specific cytotoxic T lymphocytes release gamma interferon, tumor necrosis factor alpha (TNF- $\alpha$ ), and TNF- $\beta$ when they encounter their target antigens. J. Virol. 67, 2844-2852 (1993).

16. Lohman, B. L. et al. A partially attenuated simian immunodeficiency virus induces host immunity that correlates with resistance to pathogenic virus challenge. J. Virol. 68, 7021-7029 (1994).

17. Shen, L. et al. Recombinant virus vaccine-induced SIV-specific CD8* cytotoxic T lymphocytes. Science 252, 440-443 (1991).

18. Hammond, S. A. et al. Comparative clonal analysis of human immunodeficiency virus type 1 (HIV-1)-specific CD4+ and CD8+ cytolytic T lymphocytes isolated from seronegative humans immunized with candidate HIV-1 vaccines. I. exp. Med. 176, 1531-1542 (1992).

19. Yasutomi, Y. et al. Immunization with recombinant BCG-SIV elicits SIV-specific cytotoxic $\mathrm{T}$ lymphocytes in rhesus monkeys. J. Immtun. 150, 3101-3107 (1993).

20. Aldovini, A. \& Young, R. A. Humoral and cell-mediated immune responses to live recombinant BCG-HIV vaccines. Nature 351, 479-482 (1991).

21. Miller, M. D. et al. Vaccination of rhesus monkeys with synthetic peptide in a fusogenic proteoliposome elicits simian immunodeficiency virus-specific CD8+ cytotoxic T lymphocytes. $J$. exp. Med. 176, 1739-1744 (1992).

22. Newman, M. et al. Induction of antigen-specific killer $\mathrm{T}$ lymphocyte responses using subunit SIVmac251 gag and env vaccines containing QS-21 saponin adjuvant. AIDS Res. human Retrovir 10, 853-861 (1994).

23. Wang, B. et al. Gene inoculation generates immune responses against human immunodeficiency virus type 1. Proc. natn. Acad. Sci. 90, 4156-4160 (1993).

24. Kalams, S. A. et al. Longitudinal analysis of TCR gene usage by HIV-1 envelope-specific CTL clones reveals a limited TCR repertoire. J. exp. Med. 179, 1261-1271 (1994)

Division of AIDS

National Institute of Allergy and Infectious

Diseases, National Institutes of Health

Bethesda, Maryland 20892, USA

\section{Filling in the matrix of kidney disease}

\begin{abstract}
The discovery of a gene associated with autosomal dominant polycystic kidney disease (ADPKD) was only the first step toward understanding the disease. Now researchers are determining what the gene' product normally does (pages 359-364).
\end{abstract}

Autosomal dominant polycystic kidney disease (ADPKD) affects 1 in 1,000 individuals, ROBERT BACALLAO

counseling is particularly vexmaking it one of the most common genetic diseases afflicting mankind, and the most common genetic cause of renal failure. The major manifestation of the disease is the formation of fluid filled cavities or 'cysts' within the kidney parenchyma. Often appearing first in neonates, the cysts expand over time, resulting in significantly enlarged kidneys and a host of related symptoms, including pain and bleeding episodes. The cysts also offer sanctuary to bacteria, and the resultant bacterial infections can be difficult to treat. Individuals afflicted with ADPKD face the prospect of renal failure, sudden death due to intracerebral bleeds and the possibility of passing the trait on to their children ${ }^{2}$. Genetic confidence is a computer axial tomography (CAT) scan of the kidneys using radiocontrast dye after the patient has reached age 30 (refs 2,3). Since 1957, when Dalgaard first described the disease ${ }^{4}$, there has been little to offer patients because of the paucity of therapeutic interventions and the incomplete understanding of the underlying molecular genetics.

In the past year, advances in molecular genetics have dramatically changed the outlook on ADPKD. Pioneering work that identified a $750-\mathrm{kb}$ restriction fragment length polymorphism (RFLP) from human chromosome 16 (ref. 5) led directly to the identification of the PBP (polycystic breakpoint product) gene by

The cystic kidney shown is markedly enlarged $(15 \mathrm{~cm}$ long) and the surface is studded with large fluid-filled sacs. Inset: Photomicrograph of a tissue section derived from a kidney with ADPKD.

Photographs courtesy of Frank Carone, Northwestem University Medical School, Evanston, Illinois, USA

IMAGE
UNAVAILABLE
FOR
COPYRIGHT
REASONS

